\title{
Prevalence of unknown and untreated arrhythmias in an older outpatient population screened by wireless long-term recording ECG [Corrigendum]
}

\author{
Lindberg T, Bohman DM, Elmståhl S, Jogréus C, Sanmartin
}

Berglund J. Clin Interv Aging. 2016;11:1083-1090.

On page 1086, Table 1 should be replaced by the following

table:

Table I Age distribution and medical history in the older outpatient study population

\begin{tabular}{|c|c|c|c|c|}
\hline & $\begin{array}{l}\text { Total, } \\
\text { n (\%) }\end{array}$ & $\begin{array}{l}\text { Males, } \\
\text { n (\%) }\end{array}$ & $\begin{array}{l}\text { Females, } \\
\text { n (\%) }\end{array}$ & $\begin{array}{l}P \text {-value for sex } \\
\text { differences }\end{array}$ \\
\hline Sex & $200(100)$ & $88(44.0)$ & $112(56.0)$ & n.s \\
\hline \multicolumn{5}{|l|}{ Age cohorts (years) } \\
\hline $66-80$ & $125(62.5)$ & $51(57.9)$ & $74(66.1)$ & 0.040 \\
\hline$>80$ & $75(37.5)$ & $37(42.1)$ & $38(33.9)$ & n.s \\
\hline Heart failure & II (5.5) & $3(3.4)$ & $8(7.1)$ & n.s \\
\hline Hypertension & $99(49.5)$ & $46(52.3)$ & $53(47.3)$ & n.s \\
\hline Diabetes & $9(4.5)$ & $4(4.5)$ & $5(4.4)$ & n.s \\
\hline Stroke & $13(6.5)$ & $6(6.8)$ & $7(6.2)$ & n.s \\
\hline Ischemic cardiac disease & $20(10)$ & II (I2.5) & $9(8.0)$ & n.s \\
\hline Hyper/hypothyroidism & $20(10)$ & $3(3.4)$ & $17(15.1)$ & n.s \\
\hline Smoking & $10(5)$ & $5(5.6)$ & $5(4.4)$ & n.s \\
\hline Medical treatment (eg, ASA and Warfarin) & $17(8.5)$ & II (I2.5) & $6(5.3)$ & n.s \\
\hline
\end{tabular}

Abbreviations: ASA, acetylsalicylic acid; n.s, not significant.

On page 1087, Table 2 should be replaced by the following

table:

Table 2 Heart rhythm and prevalence of arrhythmia during the analysis period in the study population

\begin{tabular}{|c|c|c|c|c|c|}
\hline & $\begin{array}{l}\text { Total }(n=200), \\
n(\%)\end{array}$ & $\begin{array}{l}95 \% \mathrm{Cl} \text { for } \\
\text { total sample }\end{array}$ & $\begin{array}{l}\text { Males }(n=88) \text {, } \\
n(\%)\end{array}$ & $\begin{array}{l}\text { Females }(n=I \mid 2) \text {, } \\
n(\%)\end{array}$ & $\begin{array}{l}P \text {-value for sex } \\
\text { differences }\end{array}$ \\
\hline Sinus rhythm & $165(82.5)$ & $77.23-87.77$ & 75 (85.2) & $90(80.4)$ & n.s \\
\hline Persistent AF & $20(10)$ & $5.84-14.16$ & $6(6.9)$ & $14(12.5)$ & n.s \\
\hline Unspecified rhythm & I $(0.5)$ & $0.0-1.48$ & $I(I . I)$ & $0(0)$ & - \\
\hline Pacemaker rhythm & $3(1.5)$ & $0.0-3.18$ & $2(2.3)$ & I (0.9) & - \\
\hline Paroxysmal AF & II (5.5) & $2.34-8.66$ & $4(4.5)$ & $7(6.2)$ & - \\
\hline $\begin{array}{l}\text { Episodes of Paroxysmal supraventricular } \\
\text { tachycardia }(>100 \text { beats } / \mathrm{min})\end{array}$ & $87(43.5)$ & $36.83-50.37$ & $33(37.5)$ & $54(48.2)$ & 0.012 \\
\hline
\end{tabular}

Abbreviations: $\mathrm{AF}$, atrial fibrillation; $\mathrm{Cl}$, confidence interval; n.s, not significant.

Clinical Interventions in Aging

\section{Publish your work in this journal}

Clinical Interventions in Aging is an international, peer-reviewed journal focusing on evidence-based reports on the value or lack thereof of treatments intended to prevent or delay the onset of maladaptive correlates of aging in human beings. This journal is indexed on PubMed Central, MedLine,

CAS, Scopus and the Elsevier Bibliographic databases. The manuscript management system is completely online and includes a very quick and fair peer-review system, which is all easy to use. Visit http://www.dovepress. com/testimonials.php to read real quotes from published authors. 